\title{
How to Evaluate Pancreaticoduodenectomy Specimens after Pancreatic Surgery? A Review
}

\author{
Nuri Emrah Goret ${ }^{1}$, Ceren Canbey Goret ${ }^{2 *}$ and Omer Faruk Ozkan ${ }^{3}$ \\ ${ }^{1}$ Department of General Surgery, Health Sciences University, Kartal Dr Lütfi Kirdar Research and Education Hospital, Istanbul, Turkey \\ ${ }^{2}$ Department of Surgical Pathology, Health Sciences University, Sancaktepe Research and Education Hospital, Istanbul, Turkey
}

${ }^{3}$ Department of General Surgery, Health Sciences University, Umraniye Research and Education Hospital, Istanbul, Turkey

Received: 眥 August 06, 2018; Published: 制 August 13, 2018

*Corresponding author: Ceren Canbey Goret, Department of Surgical Pathology, Health Sciences University, Sancaktepe Research and Education Hospital, Istanbul, Turkey

\begin{abstract}
Pancreaticoduodenectomy (Whipple procedure) is a complicated surgical procedure that is preferred in conditions such as malignancies and traumas of the pancreatic head, duodenum, bulb and choledoch or in case of pancreatitis. Careful attention should be paid to accurately determine the pathological and clinical stage of the disease in patients undergoing oncologic therapies that have changed over the years. This procedure, which is surgically troublesome, requires pathological sampling and care. During sampling, a pathologist must macroscopically analyse the pancreaticoduodenectomy specimens and carefully perform sampling.
\end{abstract}

Keywords: Whipple Procedure; Pancreas Pathology; Pancreas Gross Examination; Pancreaticoduodenectomy

\section{Introduction}

Pancreaticoduodenectomy (Whipple procedure) is a complicated surgical procedure that is preferred in conditions such as malignancies and traumas of the pancreatic head, duodenum, bulb and choledoch or in case of pancreatitis [1]. This procedure was first described by Allan Whipple in 1935 for periampullary tumours and was subsequently preferred for many other indications [2,3]. In recent years, this procedure has been highly preferred because of significant reduction in morbidity and mortality rates associated with it [4]. However, careful attention should be paid to accurately determine the pathological and clinical stage of the disease in patients undergoing oncologic therapies that have changed over the years. This procedure, which is surgically troublesome, requires pathological sampling and care. During sampling, a pathologist must macroscopically analyse the pancreaticoduodenectomy specimens and carefully perform sampling only after completely understanding the anatomical landmarks of the specimen. This will ensure that parameters essentially affecting survival, such as tumour type and lymph node status, will be evaluated more accurately [5,6] (Figures 1-4).

As reported by Adsay [7] macroscopic sampling is very important in the examination of pancreatic resection specimens.
Particularly, it is necessary to sample the entire surface of the specimen by peeling it like an orange to mark the surgical margins and obtain maximum number of lymph nodes [7]. We routinely employ this method to perform macroscopic sampling. When pancreaticoduodenectomy specimens are macroscopically examined, the pancreatic head, duodenum, distal bile duct and occasionally the distal part of the stomach and gall bladder are observed. After anatomically understanding the right, left, anterior and posterior parts of the resected specimen, the length and threedimensional measurements of all organs should be obtained. Next, surgical margins of the pancreas should be marked with ink. The surgical margins of the choledoch should be defined and sampled with a block. The duodenum should then be carefully opened from the antimesenteric border (to avoid damage to the ampulla of Vater). If there is a duodenal lesion, it should be described and measured. It is then necessary to open the main pancreatic duct and bile duct longitudinally, preferably using a stylet. The presence of enlargement/stenosis or other lesions in these areas should be reported. Then, if the stomach and gall bladder are present in the specimen, they must be opened using conventional methods, and all specimens should then be fixed in formaldehyde, preferably for 1 day, followed by macroscopic sampling. 


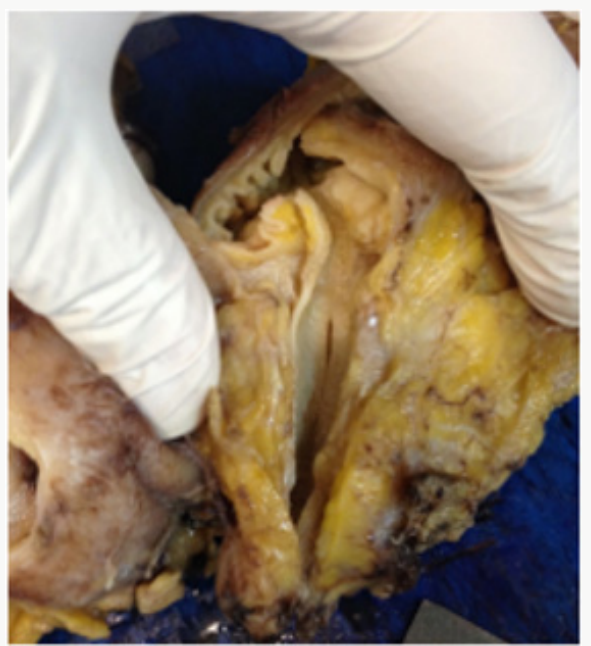

Figure 1: Mass localized in the ampulla of Vater, choledochal enlargement.

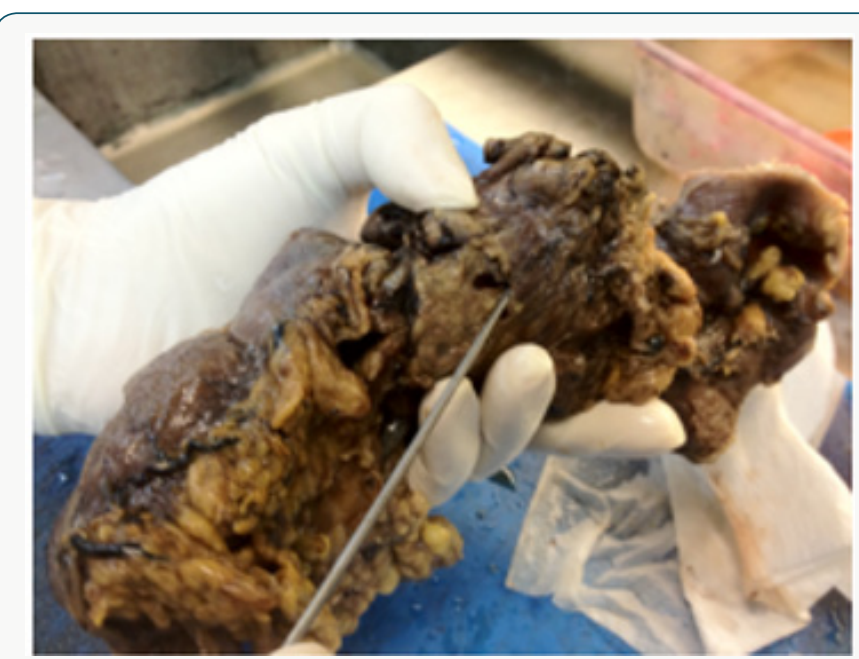

Figure 2: Mass localized in the ampulla of Vater (stile in choledoch).

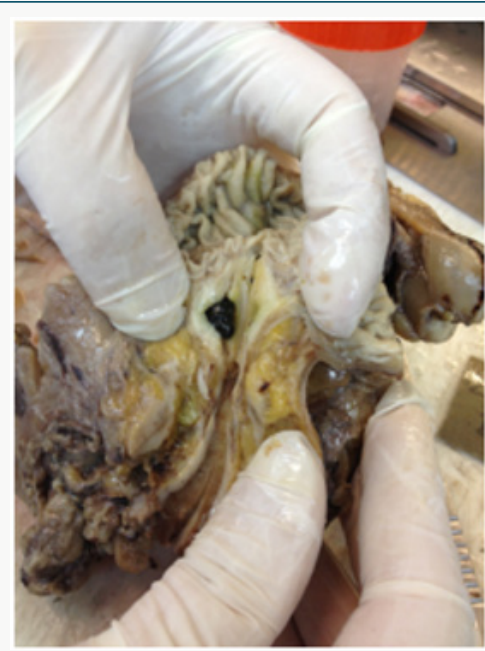

Figure 3: Pancreatitis case, stone localized in distal choledoch.

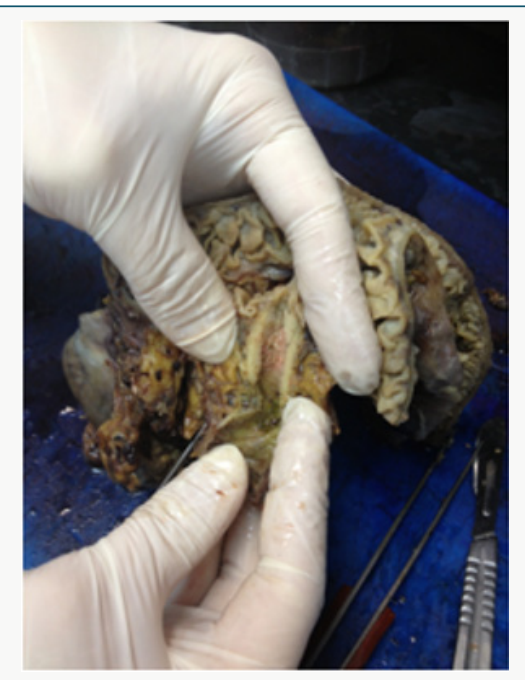

Figure 4: Mass localized in the distal choledoch and thickening of the choledoch wall.

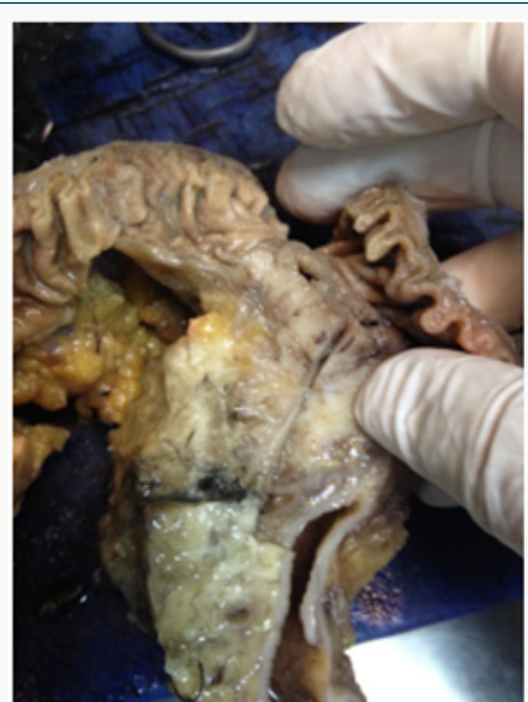

Figure 5: Mass localized in the pancreas head and tumor infiltrates the duodenum.

In pancreaticoduodenectomy specimens, the region where the tumour or lesion is present should be carefully analysed, and sampling should be performed accordingly because the behaviour and stage of tumours located in the ampulla of Vater, choledoch or pancreatic head may vary according to the area invaded by the tumours. The macroscopy report should include the association of the tumour with the duodenum, choledoch, pancreatic head, ampulla of Vater and duct of Santorini. One of the most important points in macroscopic sampling is that the distal, retroperitoneal (posterior), anterior, inferior and superior surgical margins of the pancreas should be marked and accurately sampled. In addition, after sampling all the surgical margins, the entire pancreas should be peeled like an orange and sampled after marking the margins using the method described by Adsay [7]. In addition to the surgical margins of pancreas, surgical margins of the choledoch, proximal stomach and distal duodenum should be marked and sampled $[7,8]$. 


\section{Conclusion}

In conclusion, the following points should be considered in the macroscopic examination of pancreaticoduodenectomy specimens:

a) The specimen should be anatomically analysed.

b) The duodenum should be opened from the antimesenteric border; if the stomach is included, it should be opened using the conventional method.

c) All surgical margins of the pancreas [distal, retroperitoneal (posterior), anterior, inferior and superior], choledoch, proximal stomach and distal duodenum should be accurately marked with ink and then sampled.

d) The choledoch and pancreatic duct should be opened using a stylet. The region should be described in terms of the presence of obstruction/dilation and tumour involvement.

e) The size of the tumour, its relation with anatomical structures and distance from surgical margins must be accurately described and marked, followed by tumour sampling.

f) All structures should be sampled even if there is no tumour involvement.

g) Lymph nodes dissected from the fat tissue around the stomach, duodenum and pancreas must be sampled.

\section{References}

1. Thomas B, Junginger $T$ (1999) Factors influencing morbidity and mortality after pancreaticoduodenectomy: Critical analysis of 221 resections. World J Surg 23(2): 164-172.

2. Whipple AO, Parsons WB, Mullins CR (1935) Treatment of carcinoma of the ampulla of Vater. Ann Surg 102(4): 763-779.

3. Foroughi F, Mohsenifar Z, Ahmadvand A, Zare K (2012) Pathologic findings of Whipple pancreaticoduodenectomy: a 5-year review on 51 cases at Taleghani general hospital. Gastroenterol Hepatol Bed Bench 5(4): 179-182.

4. Lillemoe KD, Kaushal S, Cameron JL, Sohn TA, Pitt HA, et al. (1999) Distal pancreatectomy: indications and outcomes in 235 patients. Ann Surg discussion 698-700 229(5): 693-698;.

5. Goret CC, Ozkan OF, Akgun MY (2017) A Rare Case Report: Synchronous Pancreatic Ductal Adenocarcinoma and Thyroid Medullary Carcinoma. Haydarpasa Numune Med J 57(2): 107-111.

6. Goret CC, Goret NE, Ozkan OF, Kilic G (2018) Clinicopathological analyses of pancreas specimens in 49 consecutive patients. Acta Medica Mediterranea 34: 1041-1045.

7. Adsay NV, Basturk O, Saka B, Bagci P, Ozdemir D, et al. (2014) Whipple made simple for surgical pathologists: orientation, dissection, and sampling of pancreaticoduodenectomy specimens for a more practical and accurate evaluation of pancreatic, distal common bile duct, and ampullary tumors. Am J Surg Pathol 38(4): 480-493.

8. Mostafa ME, Erbarut Seven I, Pehlivanoglu B, Adsay V (2017) Pathologic classification of pancreatic cancers: current concepts and challenges. Chin Clin Oncol 6(6): 59.

\section{(C) (i) This work is licensed under Creative}

To Submit Your Article Click Here: Submit Article

DOI: $10.32474 /$ LOJMS.2018.01.000104

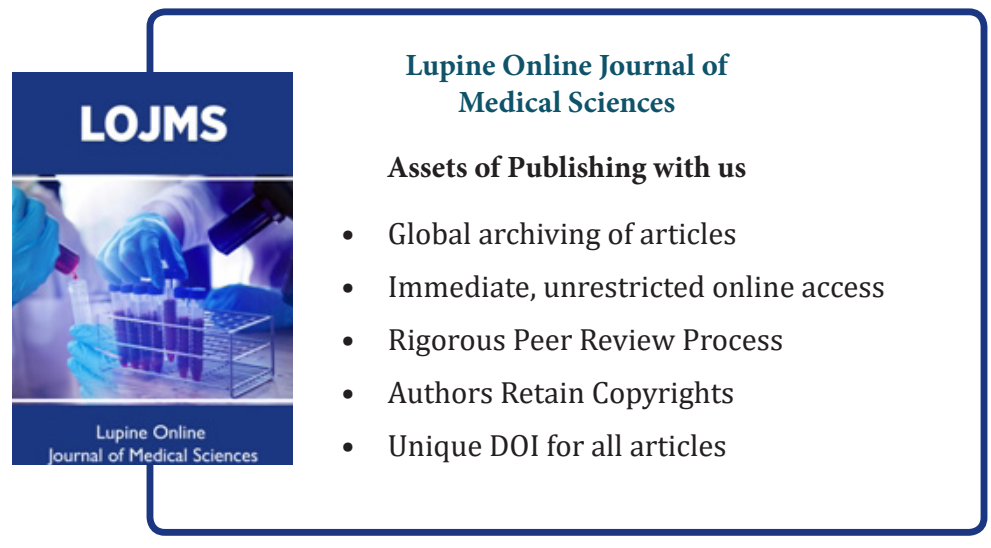

